\title{
ZENGA DISTRIBUTION AND INEQUALITY ORDERING
}

Francesco Porro

Dipartimento di Statistica e Metodi Quantitativi

Universita' degli Studi di Milano-Bicocca

Milano, Italy

francesco.porro1@unimib.it

Key Words: convex order; Lorenz order; stochastic order; Zenga index; Zenga distribution.

\begin{abstract}
The aim of this paper is to establish an ordering related to the inequality for the recently introduced Zenga distribution. In addition to the well-known order based on the Lorenz curve, the order based on $I(p)$ curve is considered. Since the Zenga distribution seems to be suitable to model wealth, financial, actuarial and especially, income distributions, these findings are fundamental in the understanding of how parameter values are related to inequality. This investigation shows that for the Zenga distribution, two of the three parameters are inequality indicators.
\end{abstract}

\section{INTRODUCTION AND PRELIMINARIES}

In a recent paper Zenga, 2010 proposed a new model for economic size distributions which can be used to model income, wealth and non-negative economic or financial variables. It depends on three non-negative parameters $\mu, \alpha$, and $\theta$ : the first one is a scale parameter and coincides with the expectation, the other two are shape parameters.

This distribution is a mixture of particular truncated Pareto distributions, analyzed by Polisicchio, 2008: these densities have the peculiarity that the corresponding inequality $I(p)$ curve is the constant line $I(p)=1-k$, with $k \in(0,1)$. The inequality $I(p)$ curve for a non-negative random variable $X$ with finite expectation and with distribution function $F$ is 
defined as

$$
I(p)=1-\frac{\bar{M}(p)}{\stackrel{+}{M}(p)} \quad p \in(0,1),
$$

where the lower mean $\bar{M}(p)$ and the upper mean $\stackrel{+}{M}(p)$ for $p \in(0,1)$, are

$$
\bar{M}(p)=\frac{1}{p} \int_{0}^{p} F^{-1}(y) d y, \text { and } \stackrel{+}{M}(p)=\frac{1}{1-p} \int_{p}^{1} F^{-1}(y) d y .
$$

In the previous formulae, $F^{-1}$ denotes the inverse function of $F$, or, in some cases, the generalized inverse function of $F$. Further detail and features of $I(p)$ curve and of the related inequality index $I$, defined by

$$
I=\int_{0}^{1} I(p) d p
$$

are in Zenga 2007. Increasing attention in the literature on the $I(p)$ curve and the index $I$ is demonstrated by the increasing number of papers on these subjects: see Polisicchio 2008, Greselin and Pasquazzi 2009, Polisicchio and Porro 2009, Radaelli 2010, Greselin et al. 2010, Porro 2011, Tille 2012, Arcagni and Porro 2012.

The mixing function on the parameter $k$ is a Beta density with non-negative parameters $\alpha$ and $\theta$. Since all the conditional densities have expectation equal to $\mu$, the expected value of the Zenga distribution is $\mu$.

The model is characterized by the probability density function:

$$
f(x ; \mu, \alpha, \theta)= \begin{cases}\frac{1}{2 \mu B(\alpha, \theta)}\left(\frac{x}{\mu}\right)^{-3 / 2} \int_{0}^{\frac{x}{\mu}} k^{\alpha-1 / 2}(1-k)^{\theta-2} d k & \text { for } 0<x \leq \mu \\ \frac{1}{2 \mu B(\alpha, \theta)}\left(\frac{\mu}{x}\right)^{3 / 2} \int_{0}^{\frac{\mu}{x}} k^{\alpha-1 / 2}(1-k)^{\theta-2} d k & \text { for } x>\mu,\end{cases}
$$

where $B(a, b)$ denotes the Beta function, related to the Gamma function by $B(a, b)=$ $\Gamma(a) \Gamma(b) / \Gamma(a+b)$, for any $a>0$ and $b>0$.

This density function is very flexible and its plot can have different shapes depending on the values of the parameters. The Figures 1 and 2 show a selection of them with the corresponding distribution functions. 

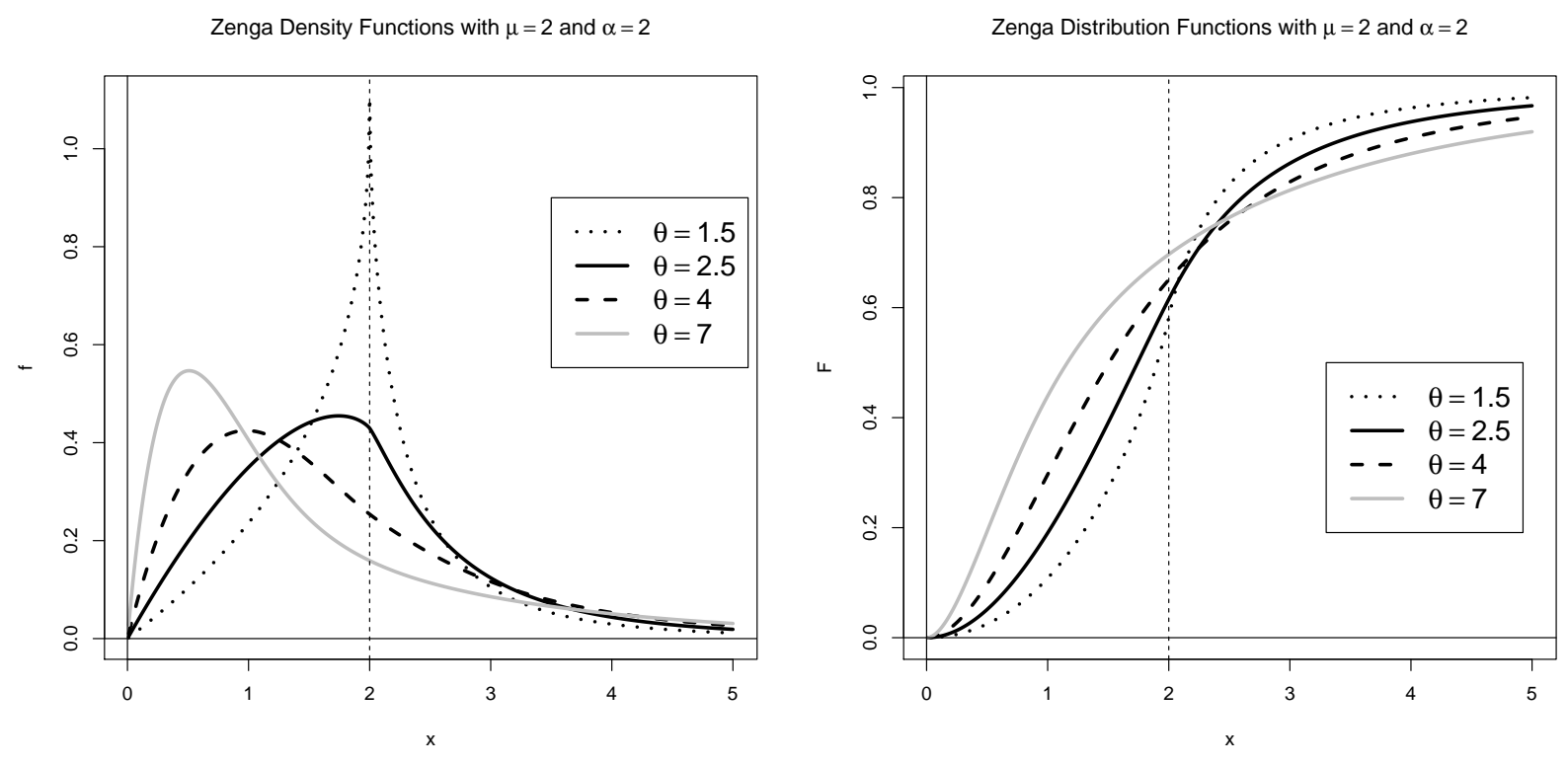

Figure 1: Density (on the left) and distribution (on the right) functions of the Zenga model with $\mu=2$ and $\alpha=2$

Some features, like the finite expectation for any admissible choice of the parameters, the positive skewness, the existence (under restrictions on parameter $\alpha$ ) of the moments, and the Paretian right tail, have already been investigated in Zenga, 2010 and in Zenga et al., 2011: all these characteristics make this distribution interesting for methodological studies and for applications as well.

This paper aims to analyse the role of the parameters of the Zenga distribution in terms of inequality. In the literature, many papers perform the same analysis for other families of distributions: Taillie (1981) deals with the generalized gamma, Wilfling and Kramer (1993) with the Singh-Maddala, Wilfling (1996) and Kleiber (1999) with the generalized Beta of II kind, and Sarabia et al. (2003) with the Mcdonalds generalized functions.

It is well-known that for many distribution families, depending on one parameter (aside from the scale parameter), the corresponding Lorenz curves do not intersect. Hence, for such families, the order based on the Lorenz curve and hence also the order based on the $I(p)$ curve are linear. Even if this allows the comparison in terms of inequality between any two elements 

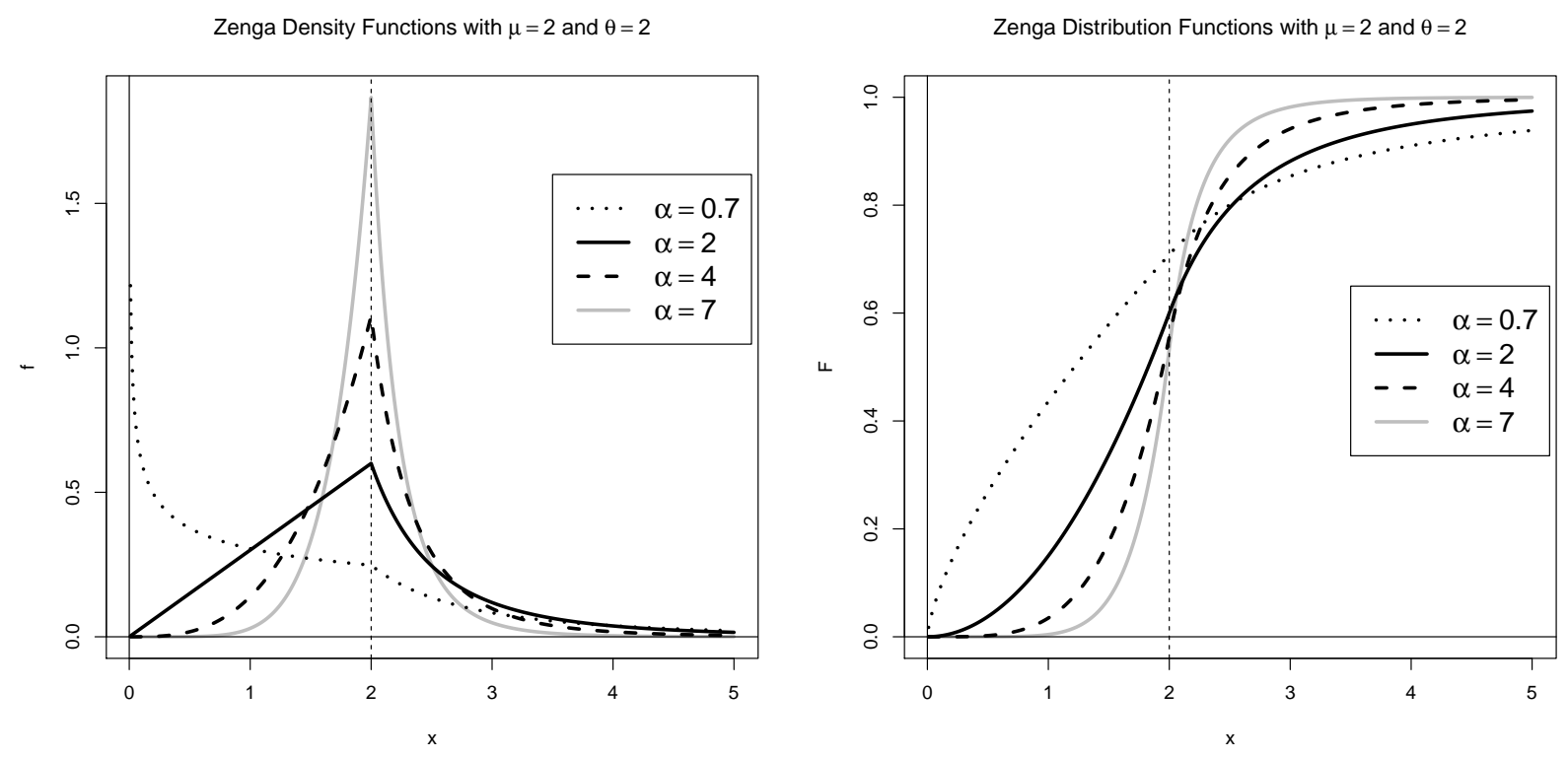

Figure 2: Density (on the left) and distribution (on the right) functions of the Zenga model with $\mu=2$ and $\theta=2$

of the family, Taillie (1981) claims that, in some way, the use of such single-parameter families "...would impose upon the fitted curves structural and comparative features not present in the data". A solution to overcome this restriction is the use of distribution families with more than one parameter, such as the Zenga distribution. In the present analysis, $\mu$ is excluded, since it is a scale parameter, and it therefore does not affect the inequality. So, the focus is on the two remaining parameters $\alpha$ and $\theta$.

This paper is outlined as follows. The definitions of the considered partial orders and their relations are provided in Section 2. In Section 3 the main findings regarding the partial order based on the Lorenz curve are stated and proved. Section 4 is devoted to the extension to the partial order based on $I(p)$ curve. Section 5 describes an application to real income data. Finally, some remarks and the conclusion are in the last Section.

\section{THE ORDERINGS}

This paper considers and compares three partial orderings. The first one is based on the 
Lorenz curve $\left(\leq_{L}\right)$ and it is the most famous and classical ordering for measuring inequality. It is strictly related to the Lorenz curve, since two distributions are comparable with respect to this ordering if the corresponding Lorenz curves do not intersect.

Definition 1 Let $X_{1}$ and $X_{2}$ be two continuous non-negative random variables with finite and positive expectations. Let $L_{X_{1}}$ and $L_{X_{2}}$ denote their Lorenz curves. Then: $X_{1} \geq_{L} X_{2} \Leftrightarrow$ $L_{X_{1}}(p) \leq L_{X_{2}}(p), \forall p \in(0,1)$.

The second ordering considered here is based on the inequality $I(p)$ curve $\left(\leq_{I}\right)$, which appeared recently in the literature. Similarly to the previous case, two distributions are comparable with respect to this ordering if the corresponding $I(p)$ curves do not intersect.

Definition 2 Let $X_{1}$ and $X_{2}$ be two continuous non-negative random variables with finite and positive expectations. Let $I_{X_{1}}$ and $I_{X_{2}}$ denote their inequality $I(p)$ curves. Then: $X_{1} \geq_{I}$ $X_{2} \Leftrightarrow I_{X_{1}}(p) \geq I_{X_{2}}(p), \forall p \in(0,1)$.

The third and last ordering is the so called convex ordering $\left(\leq_{C X}\right)$, which claims that two distributions are comparable if they are comparable according to the criterion of the expected utility, assuming that the utility function is increasing and concave. It is worthy to note that a concave utility function characterizes a risk averse agent.

Definition 3 Let $X_{1}$ and $X_{2}$ be two continuous non-negative random variables with finite and positive expectations. Then: $X_{1} \geq_{C X} X_{2} \Leftrightarrow \mathbb{E}\left[\phi\left(X_{1}\right)\right] \geq \mathbb{E}\left[\phi\left(X_{2}\right)\right]$ for all convex functions $\phi: \mathbb{R} \rightarrow \mathbb{R}$, such that the expectations exist.

For further detail and features refer to Shaked and Shanthikumar 2007, Polisicchio and Porro 2009, Atkinson 1970. If the two random variables have the same expectation, as claimed in the following proposition, the three orders are equivalent.

Proposition 1 Let $X_{1}$ and $X_{2}$ be two continuous non-negative random variables with the same finite and positive expectation. Then the following three assertions are equivalent:

i) $X_{2} \leq_{C X} X_{1}$; 
ii) $X_{2} \leq_{L} X_{1}$;

iii) $X_{2} \leq_{I} X_{1}$.

Proof that $i) \Leftrightarrow i$ ) can be found in Shaked and Shanthikumar 2007, while proof that $i i) \Leftrightarrow$ iii) is in Polisicchio and Porro 2009. Proposition 1 is very useful in the following sections.

\section{RESULTS RELATED TO THE ORDER BASED ON THE LORENZ CURVE}

Before stating the two main Propositions, which are the core contribution of this paper, two useful lemmas are needed.

Lemma 1 Let $X_{1}$ and $X_{2}$ be two continuous random variables with the same expectation and probability density function $f_{1}$ and $f_{2}$, respectively. If the function $f_{2}-f_{1}$ changes sign twice and the sign sequence is,,+-+ , then $X_{1} \leq_{C X} X_{2}$.

For a proof, see Shaked and Shanthikumar, 2007.

Lemma 2 Let $y$ be a positive constant. Then the function $h_{y}(x)=\Gamma(x) / \Gamma(x+y), x>0$ is monotonically strictly decreasing in $x$.

\section{Proof}

The derivative of the function $h_{y}$ with respect to $x$ is:

$$
\frac{d h_{y}(x)}{d x}=\frac{\Gamma(x) \Gamma(x+y)}{[\Gamma(x+y)]^{2}}[\psi(x)-\psi(x+y)],
$$

where $\psi$ is the digamma function $\psi(x)=\Gamma^{\prime}(x) / \Gamma(x)$. It is well-known that for $x>0$, the digamma function $\psi(x)$ is monotonically increasing, see Abramowitz and Stegun, 1964. This implies that $d h_{y}(x) / d x<0$ and therefore $h_{y}(x)$ is a monotonically decreasing function.

Now, the first proposition regarding the ordering of the Zenga distribution can be stated and proved. In the following, $X \sim \operatorname{Zenga}(\mu, \alpha, \theta)$ will denote that the distribution of the random variable $X$ has a Zenga distribution with parameters $\mu, \alpha, \theta$. 
Proposition 2 Let $X_{1}$ and $X_{2}$ be two continuous random variables such that $X_{1} \sim$ Zenga $\left(\mu, \alpha_{1}, \theta\right)$ and $X_{2} \sim Z$ Zenga $\left(\mu, \alpha_{2}, \theta\right)$, where $0<\alpha_{1}<\alpha_{2}, \theta>0$ and $\mu>0$. Then $X_{2} \leq_{L} X_{1}$.

\section{Proof}

Note that, by hypothesis, the random variables $X_{1}$ and $X_{2}$ own the same expected value $\mu$. Now, let $f_{i}$ denote the probability density function of $X_{i}, i=1,2$. In order to apply Lemma 1 , the sign changes of $f_{2}-f_{1}$ have to be studied. Let $g$ be the ratio of the two density functions: $g(x)=f_{1}(x) / f_{2}(x)$. In $(0, \mu)$ and in $(\mu,+\infty) g$ is continuous, since $f_{1}$ and $f_{2}$ are continuous.

Consider the case $x \in(0, \mu)$ first. The behavior of $g$ around the extreme values of this interval is described by the two limits that can be evaluated using the De l'Hospital's rule:

$$
\begin{gathered}
\lim _{x \rightarrow 0^{+}} g(x)=\lim _{x \rightarrow 0^{+}} \frac{B\left(\alpha_{2}, \theta\right)}{B\left(\alpha_{1}, \theta\right)} \frac{\int_{0}^{x / \mu} k^{\alpha_{1}-1 / 2}(1-k)^{\theta-2} d k}{\int_{0}^{x / \mu} k^{\alpha_{2}-1 / 2}(1-k)^{\theta-2} d k}=\lim _{x \rightarrow 0^{+}} \frac{B\left(\alpha_{2}, \theta\right)}{B\left(\alpha_{1}, \theta\right)}\left(\frac{x}{\mu}\right)^{\alpha_{1}-\alpha_{2}}=+\infty \\
\lim _{x \rightarrow \mu^{-}} g(x)= \begin{cases}\frac{\Gamma\left(\alpha_{2}\right) \Gamma\left(\alpha_{2}+\theta-1 / 2\right) \Gamma\left(\alpha_{1}+1 / 2\right) \Gamma\left(\alpha_{1}+\theta\right)}{\Gamma\left(\alpha_{2}+1 / 2\right) \Gamma\left(\alpha_{2}+\theta\right) \Gamma\left(\alpha_{1}\right) \Gamma\left(\alpha_{1}+\theta-1 / 2\right)} & \text { if } \theta>1 \\
\frac{\Gamma\left(\alpha_{2}\right) \Gamma\left(\alpha_{1}+\theta\right)}{\Gamma\left(\alpha_{2}+\theta\right) \Gamma\left(\alpha_{1}\right)} & \text { if } 0<\theta \leq 1\end{cases}
\end{gathered}
$$

For Lemma 2, it follows that $\lim _{x \rightarrow \mu^{-}} g(x)<1$ for any positive value of $\theta$.

The derivative of function $g$ is:

$$
\frac{d g(x)}{d x}=c \cdot \int_{0}^{x / \mu}(1-k)^{\theta-2} k^{\alpha_{1}-1 / 2}\left(\frac{x}{\mu}\right)^{\alpha_{1}-1 / 2}\left[k^{\alpha_{2}-\alpha_{1}}-\left(\frac{x}{\mu}\right)^{\alpha_{2}-\alpha_{1}}\right] d k
$$

where

$$
c=\frac{B\left(\alpha_{2}, \theta\right)}{\mu B\left(\alpha_{1}, \theta\right)}\left(1-\frac{x}{\mu}\right)^{\theta-2}\left[\int_{0}^{x / \mu} k^{\alpha_{2}-1 / 2}(1-k)^{\theta-2} d k\right]^{-2}>0 .
$$

The integrand function in equation (3) is negative for $k \in(0, x / \mu)$, therefore the value of the integral is strictly smaller than 0 . This means that $d g(x) / d x$ is negative and then $g$ is a monotonically strictly decreasing function in $(0, \mu)$. The aforementioned considered limits and the monotonicity imply that there exists a unique $x_{0} \in(0, \mu)$ such that $g\left(x_{0}\right)=$ 
$f_{1}\left(x_{0}\right) / f_{2}\left(x_{0}\right)=1$, and equivalently $f_{2}\left(x_{0}\right)-f_{1}\left(x_{0}\right)=0$. This means that the function $f_{2}-f_{1}$ changes sign only once in the interval $(0, \mu)$ with the sign sequence:,-+ .

Now, let $x$ belong to $(\mu,+\infty)$. As before, using the De l'Hospital's rule, the limits of the function $g$ as $x$ approaches to the extreme values of this new interval are

$$
\lim _{x \rightarrow \mu+} g(x)= \begin{cases}\frac{\Gamma\left(\alpha_{2}\right) \Gamma\left(\alpha_{2}+\theta-1 / 2\right) \Gamma\left(\alpha_{1}+1 / 2\right) \Gamma\left(\alpha_{1}+\theta\right)}{\Gamma\left(\alpha_{2}+1 / 2\right) \Gamma\left(\alpha_{2}+\theta\right) \Gamma\left(\alpha_{1}\right) \Gamma\left(\alpha_{1}+\theta-1 / 2\right)} & \text { for } \theta>1 \\ \frac{\Gamma\left(\alpha_{2}\right) \Gamma\left(\alpha_{1}+\theta\right)}{\Gamma\left(\alpha_{2}+\theta\right) \Gamma\left(\alpha_{1}\right)} & \text { for } 0<\theta \leq 1,\end{cases}
$$

and

$$
\lim _{x \rightarrow+\infty} g(x)=\lim _{x \rightarrow+\infty} \frac{B\left(\alpha_{2}, \theta\right)}{B\left(\alpha_{1}, \theta\right)}\left(\frac{x}{\mu}\right)^{\alpha_{2}-\alpha_{1}}=+\infty .
$$

Since limit (4) coincides with the previous limit $(2), \lim _{x \rightarrow \mu^{+}} g(x)<1$ for any positive value of parameter $\theta$.

The derivative of function $g$ in the interval $(\mu,+\infty)$ is:

$$
\frac{d g(x)}{d x}=a \cdot \int_{0}^{\mu / x}(1-k)^{\theta-2} k^{\alpha_{1}-1 / 2}\left(\frac{\mu}{x}\right)^{\alpha_{1}-1 / 2}\left[k^{\alpha_{2}-\alpha_{1}}-\left(\frac{\mu}{x}\right)^{\alpha_{2}-\alpha_{1}}\right] d k,
$$

where

$$
a=\frac{B\left(\alpha_{2}, \theta\right)}{B\left(\alpha_{1}, \theta\right)}\left(1-\frac{\mu}{x}\right)^{\theta-2}\left(-\frac{\mu}{x^{2}}\right)\left[\int_{0}^{\mu / x} k^{\alpha_{2}-1 / 2}(1-k)^{\theta-2} d k\right]^{-2}<0 .
$$

Similarly to the previous case, the integrand function in equation (5) is negative for $k \in$ $(0, \mu / x)$, therefore the value of the integral is strictly smaller than 0 ; hence $d g(x) / d x$ is positive and then $g$ is a monotonically increasing function in $(\mu,+\infty)$. Hence there exists a unique $x_{0} \in(\mu,+\infty)$ such that $g\left(x_{0}\right)=f_{1}\left(x_{0}\right) / f_{2}\left(x_{0}\right)=1$, and equivalently $f_{2}\left(x_{0}\right)-f_{1}\left(x_{0}\right)=$ 0 . Thus, there exists only one sign change of the function $f_{2}-f_{1}$ for $x \in(\mu,+\infty)$ and the sign sequence is,+- .

The results related to the two intervals $(0, \mu)$ and $(\mu,+\infty)$ guarantee that the function $f_{2}-f_{1}$ has only two sign changes, with sign sequence,,-+- , hence for Lemma 1 , it holds that $X_{2} \leq_{C X} X_{1}$. Finally, applying Proposition 1, it follows that $X_{2} \leq_{L} X_{1}$. 
The second proposition regarding the ordering of the Zenga distribution is stated and proved in the following.

Proposition 3 Let $X_{1}$ and $X_{2}$ be two continuous random variables such that $X_{1} \sim$ Zenga $\left(\mu, \alpha, \theta_{1}\right)$ and $X_{2} \sim$ Zenga $\left(\mu, \alpha, \theta_{2}\right)$, where $0<\theta_{1}<\theta_{2}, \alpha>0$ and $\mu>0$. Then $X_{1} \leq_{L} X_{2}$.

\section{Proof}

The plan of proof is very similar to the one of Proposition 2. Also in this case the ratio $g$ of the two probability density functions $f_{i}$ of $X_{i}(i=1,2)$ is considered: $g(x)=f_{1}(x) / f_{2}(x)$.

The behavior of function $g$ as $x$ tends to 0 and to infinity is described by the two limits

$$
\lim _{x \rightarrow 0^{+}} g(x)=\frac{\Gamma\left(\theta_{2}\right) \Gamma\left(\alpha+\theta_{1}\right)}{\Gamma\left(\alpha+\theta_{2}\right) \Gamma\left(\theta_{1}\right)} \text { and } \lim _{x \rightarrow+\infty} g(x)=\frac{\Gamma\left(\theta_{2}\right) \Gamma\left(\alpha+\theta_{1}\right)}{\Gamma\left(\alpha+\theta_{2}\right) \Gamma\left(\theta_{1}\right)},
$$

which, for Lemma 2 are smaller than 1 . As $x$ tends to $\mu$ :

$$
\lim _{x \rightarrow \mu} g(x)= \begin{cases}\frac{\Gamma\left(\theta_{2}\right) \Gamma\left(\alpha+\theta_{1}\right) \Gamma\left(\theta_{1}-1\right) \Gamma\left(\alpha+\theta_{2}-1 / 2\right)}{\Gamma\left(\alpha+\theta_{2}\right) \Gamma\left(\theta_{1}\right) \Gamma\left(\alpha+\theta_{1}-1 / 2\right) \Gamma\left(\theta_{2}-1\right)} & \text { for } \theta_{1}>1 \\ +\infty & \text { otherwise }\end{cases}
$$

since for $0<\theta_{2} \leq 1$, by De l'Hospital's rule it holds that

$$
\begin{aligned}
& \lim _{x \rightarrow \mu^{-}} g(x)=\lim _{x \rightarrow \mu^{-}} \frac{\Gamma\left(\theta_{2}\right) \Gamma\left(\alpha+\theta_{1}\right)}{\Gamma\left(\alpha+\theta_{2}\right) \Gamma\left(\theta_{1}\right)}\left(1-\frac{x}{\mu}\right)^{\theta_{1}-\theta_{2}}=+\infty \\
& \lim _{x \rightarrow \mu^{+}} g(x)=\lim _{x \rightarrow \mu^{+}} \frac{\Gamma\left(\theta_{2}\right) \Gamma\left(\alpha+\theta_{1}\right)}{\Gamma\left(\alpha+\theta_{2}\right) \Gamma\left(\theta_{1}\right)}\left(1-\frac{\mu}{x}\right)^{\theta_{1}-\theta_{2}}=+\infty,
\end{aligned}
$$

while for $0<\theta_{1} \leq 1$ and $\theta_{2}>1$,

$$
\lim _{x \rightarrow \mu} g(x)=\lim _{x \rightarrow \mu} \frac{\Gamma\left(\alpha+\theta_{2}-1 / 2\right)}{\Gamma(\alpha+1 / 2) \Gamma\left(\theta_{2}-1\right)} \cdot \int_{0}^{x / \mu} k^{\alpha-1 / 2}(1-k)^{\theta_{1}-2} d k=+\infty .
$$

For Lemma 2, $\lim _{x \rightarrow \mu^{ \pm}} g(x)>1$ for any positive values of $\theta_{1}$ and $\theta_{2}$. Through an analysis of the sign of the derivative, it is possible to show that the function $g$ is strictly monotonic increasing in the interval $(0, \mu)$, and strictly monotonic decreasing in $(\mu,+\infty)$. The procedure is the same as the one of Proposition 2, since, for $x \in(0, \mu)$ :

$$
\frac{d g(x)}{d x}=z \cdot \int_{0}^{x / \mu} k^{\alpha-1 / 2}(1-k)^{\theta_{1}-2}\left(1-\frac{x}{\mu}\right)^{\theta_{1}-2}\left[(1-k)^{\theta_{2}-\theta_{1}}-\left(1-\frac{x}{\mu}\right)^{\theta_{2}-\theta_{1}}\right] d k,
$$


where

$$
z=\frac{B\left(\alpha, \theta_{2}\right)}{\mu B\left(\alpha, \theta_{1}\right)}\left(\frac{x}{\mu}\right)^{\alpha-1 / 2}\left[\int_{0}^{x / \mu} k^{\alpha-1 / 2}(k)^{\theta_{2}-2} d k\right]^{-2}>0
$$

while for $x \in(\mu,+\infty)$ :

$$
\frac{d g(x)}{d x}=w \cdot \int_{0}^{\mu / x} k^{\alpha-1 / 2}(1-k)^{\theta_{1}-2}\left(1-\frac{\mu}{x}\right)^{\theta_{1}-2}\left[(1-k)^{\theta_{2}-\theta_{1}}-\left(1-\frac{\mu}{x}\right)^{\theta_{2}-\theta_{1}}\right] d k
$$

with

$$
w=\frac{B\left(\alpha, \theta_{2}\right)}{B\left(\alpha, \theta_{1}\right)}\left(\frac{\mu}{x}\right)^{\alpha-1 / 2}\left(-\frac{\mu}{x}\right)\left[\int_{0}^{\mu / x} k^{\alpha-1 / 2}(k)^{\theta_{2}-2} d k\right]^{-2}<0 .
$$

Hence $d g(x) / d x>0$ if $x \in(0 ; \mu)$, and $d g(x) / d x<0$ in $(\mu,+\infty)$.

As in Proposition 2, it can be stated that:

- in $(0, \mu)$ there exists only one point where the function $f_{2}-f_{1}$ changes sign, and the sign sequence is,+- ;

- in $(\mu,+\infty)$ there exists only one point where the function $f_{2}-f_{1}$ changes sign, and the sign sequence is,-+ ,

hence $f_{2}-f_{1}$ changes sign only twice with sign sequence,,+-+ and for Lemma 1 it follows that $X_{1} \leq_{C X} X_{2}$. As in Proposition 2, the thesis can easily be achieved, using Proposition 1.

Since for the Zenga distribution $\mu$ is a scale-parameter, the previous two ordering propositions can be combined, leading to the following theorem.

Theorem 1 Let $X_{1}$ and $X_{2}$ be two continuous random variables such that $X_{i} \sim$ Zenga $\left(\mu_{i}, \alpha_{i}, \theta_{i}\right)$, where $\theta_{i}>0, \alpha_{i}>0$ and $\mu_{i}>0$, for $i=1,2$. If $\alpha_{1} \leq \alpha_{2}$ and $\theta_{1} \geq \theta_{2}$, then $X_{2} \leq_{L} X_{1}$.

In this theorem, the two random variables $X_{1}$ and $X_{2}$ can have different means, since the order based on the Lorenz curve does not depend on scale parameters. In effect, if $X_{1}$ and $X_{2}$ are two random variables satisfying the hypothesis of Theorem 1 , consider $Y_{1}=X_{1} / \mu_{1}$ and $Y_{2}=X_{2} / \mu_{2}$. Through Propositions 2 and 3 , it follows that $Y_{2} \leq_{L} Y_{1}$. But $X_{1}$ and $X_{2}$ have the same Lorenz curve of $Y_{1}$ and $Y_{2}$, respectively: it therefore also holds true that $X_{2} \leq_{L} X_{1}$. 
Theorem 1 also highlights a result related to the well-known Gini index, the most famous inequality index defined by:

$$
G=2 \int_{0}^{1}[p-L(p)] d p
$$

where $L(p)$ is the Lorenz curve. Since the order based on the Lorenz curve implies the same ordering in terms of the Gini index, for two random variables $X_{1}$ and $X_{2}$ satisfying the hypothesis of Theorem 1, the relation between their Gini indices is $G_{X_{2}} \leq G_{X_{1}}$.

\section{EXTENSION TO THE ORDER BASED ON I(P) CURVE}

As stated before, since Proposition 1 holds, the results of the previous section related to the order based on $L(p)$ curve can be easily extended to the partial order based on $I(p)$ curve. This means that the two previous ordering propositions can be stated also for the order based on $I(p)$ curve: the only change is to replace $\leq_{L}$ with $\leq_{I}$. Hence, under the assumptions of Proposition 2, it holds that $X_{2} \leq_{I} X_{1}$, and under the assumptions of Proposition 3, it holds that $X_{1} \leq_{I} X_{2}$. For the sake of completeness, the following summarizing corollary regarding the order based on $I(p)$ curve is reported.

Corollary 1 Let $X_{1}$ and $X_{2}$ be two continuous random variables such that $X_{i} \sim \operatorname{Zenga}\left(\mu_{i}, \alpha_{i}, \theta_{i}\right)$, where $\theta_{i}>0, \alpha_{i}>0$ and $\mu_{i}>0$, for $i=1,2$. If $\alpha_{1} \leq \alpha_{2}$ and $\theta_{1} \geq \theta_{2}$, then $X_{2} \leq_{I} X_{1}$.

This corollary can be clearly interpreted from a geometrical point of view. It states that an inverse variation of the parameters $\alpha$ and $\theta$ in the Zenga distribution, implies that the corresponding $I(p)$ curves are ordered: they do not cross each other and one lies below the other.

Let $I_{X}$ denote the index $I$ of the distribution $X$. By the definition of the inequality index $I$ (see equation (1)), if $X_{1}$ and $X_{2}$ are two random variables satisfying the hypothesis of Corollary 1, then $I_{X_{2}} \leq I_{X_{1}}$. Hence, Corollary 1 provides the ranking also between the corresponding indices.

\section{AN APPLICATION}

Table 1 shows the parameter estimates and two inequality indexes (Gini and $I$ ) for the fitted models, obtained from three real income distributions, see Arcagni and Porro, 2012. 
The sources of the data are:

- Italy 2006 Household income (Bank of Italy, 2008), with 7,762 observations;

- Switzerland 2005 Household income (Swiss Federal Statistical Office, 2005), with 3,071 observations;

- USA 2008 Household income (U.S. Census Bureau Data Ferret, 2008), with 2,899,458 observations.

Refer to Arcagni and Porro, 2012 for more details regarding the dataset and for the method of parameter estimation.

\begin{tabular}{lcccccc}
\hline \hline & \multicolumn{3}{c}{ Estimates } & & \multicolumn{2}{c}{ Inequality } \\
\cline { 2 - 4 } \cline { 7 - 8 } & $\hat{\mu}$ & $\hat{\alpha}$ & $\hat{\theta}$ & & Gini & $I$ \\
\hline Italy 2006 & 31918.93 & 2.4447 & 4.0653 & & 0.3430 & 0.6837 \\
Switzerland 2005 & 6783.75 & 4.0210 & 4.8195 & & 0.3042 & 0.6392 \\
USA 2008 & 82460.21 & 3.9922 & 10.7071 & & 0.4479 & 0.7913 \\
\hline \hline
\end{tabular}

Table 1: Parameter estimations and inequality indexes for three real income distributions

The three distributions can be ranked in terms of their Gini index (or equivalently in terms of their inequality index $I$ ), but Theorem 1 can be applied only for the comparison between the USA and the Swiss distributions. This means that the models fitted to USA and Swiss incomes have non-intersecting Lorenz curves, therefore the model fitted to USA incomes is more unequal than the model fitted to Swiss data for all the Lorenz-consistent inequality measures. Regarding all the other pairwise comparisons, Theorem 1 can not be applied, therefore it does not provide information on the ranking of the Lorenz curves for the fitted distributions. Figure 3 shows the Lorenz and the $I(p)$ curves of the fitted distributions. It is worthy to remark that the curves of Switzerland and Italy do not intersect, while the curves of USA and Italy cross each other in a neighborhood of zero. In both of such cases, Theorem 1 does not apply. 


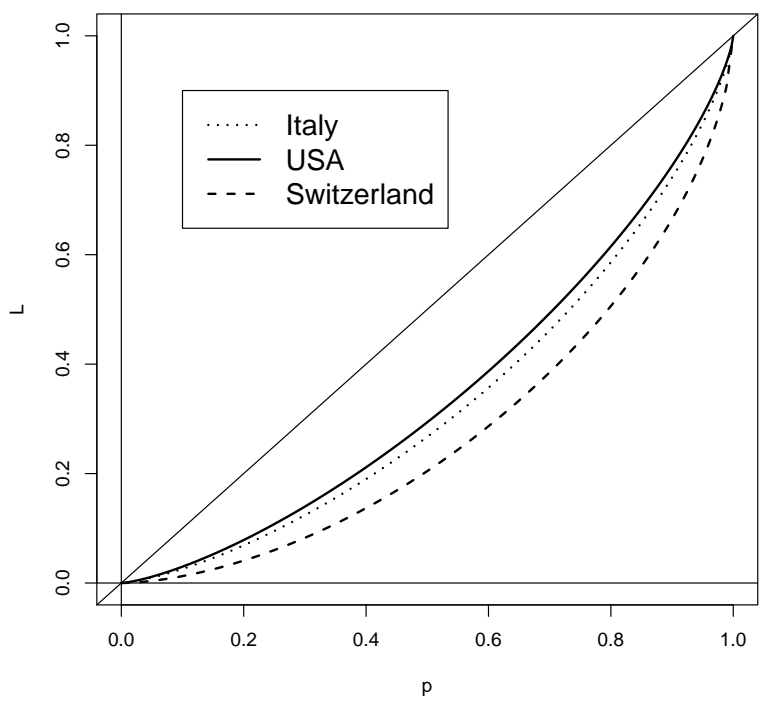

I(p) curves

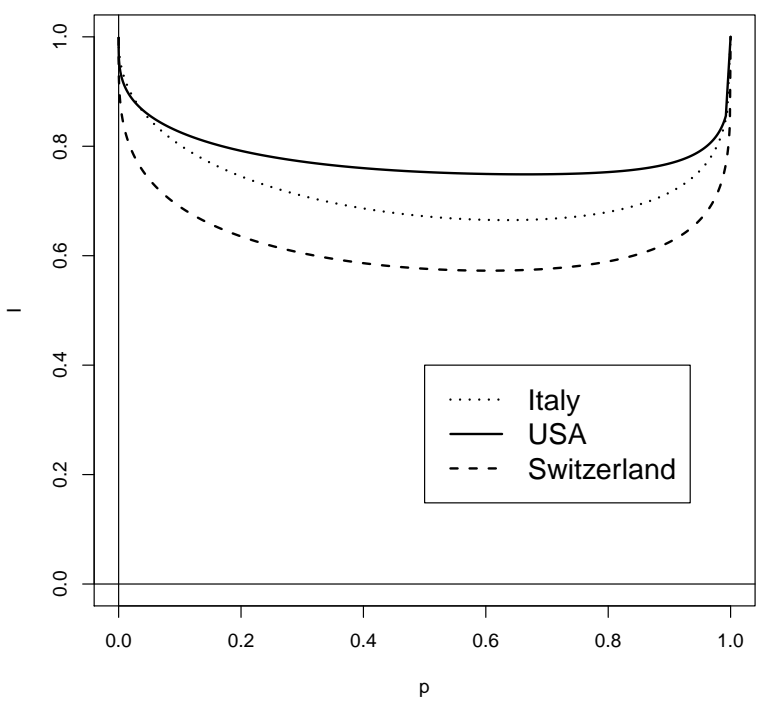

Figure 3: The $L(p)$ curves (on the left) and the $I(p)$ curves (on the right) for the three fitted income distributions

\section{CONCLUSIONS}

This paper shows that for the Zenga distribution, the Lorenz and the $I(p)$ curves are nested as one shape parameter changes. In detail, they prove that $\alpha$ is an inverse inequality indicator, and $\theta$ is a direct inequality indicator for these two curves. The bigger the $\alpha$ value, the less unequal the distribution and the bigger the $\theta$ value, the more unequal the distribution. Moreover the case where both the shape parameters change is also approached: an inverse variation of them leads to an ordering of the considered inequality curves. Obviously, also the Gini index and the Zenga index $I$, being related to these two curves, inherit the same ordering. The third parameter of the Zenga distribution, $\mu$ is a scale parameter and hence it does not modify the inequality at all.

\section{BIBLIOGRAPHY}

Abramowitz, M., Stegun, I. (1964). Handbook of mathematical functions. Dover, New York. 
Arcagni, A, Porro, F. (2012). On the parameters of Zenga distribution. Statistical Methods and Applications - doi:.1007/s10260-012-0219-y.

Atkinson, A.B. (1970). On the measurement of inequality. Journal of Economic Theory, 2, $244-263$.

Banca d'Italia (2008). The 2006 Bank of Italy sample survey on househlod income and wealth. Available at http://www.bancaditalia.it

Greselin, F., Pasquazzi, L. (2009). Asymptotic confidence intervals for a new inequality measure. Communications in Statistics - Simulation and Computation, 38, 1742-1756.

Greselin, F., Pasquazzi, L., Zitikis, R. (2010). Zenga's new index of economic inequality, its estimation, and an analysis of incomes in Italy. J. Probab. Stat., vol. 2010, ID 718905 1-26.

Kleiber, C. (1999). On the Lorenz order within parametric families of income distributions. Sankhya B, 61, 514-517.

Langel, M., Tillé, Y. (2011). Inference by linearization for Zenga's new inequality index: a comparison with the Gini index. Metrika, 75, 1093-1110.

Polisicchio, M. (2008). The continuous random variable with uniform point inequality measure I(p). Statistica \& Applicazioni, 6, 137-151.

Polisicchio, M., Porro, F. (2009). A comparison between Lorenz L(p) curve and Zenga I(p) curve. Statistica Applicata - Italian Journal of Applied Statistics, 21, 289-301.

Porro, F. (2011). The distribution model with linear inequality curve I(p). Statistica \& Applicazioni, 9, 47-61.

Radaelli, P. (2010). On the decomposition by subgroups of the Gini index and Zenga's uniformity and inequality indexes. International Statistical Review, 78, 81-101.

Sarabia, J.M., Castillo, E., Slottje, D.E. (2002). Lorenz ordering between Mcdonalds generalized functions of the income size distribution. Econom. Lett., 75, 265-270. 
Shaked, M., Shanthikumar, J.G. (2007). Stochastic Orders. Springer, New York.

Swiss Federal Statistical Office(FSO) (2005). Household Budget Survey (HBS)

(EBM/HABE/IBED). Available at http://www.bfs.admin.ch

U.S. Census Bureau Data Ferret (2008). American Community Survey (ACS2008). Available at http://www.census.gov/acs/www/

Taillie, C. (1981). Lorenz ordering within the generalized gamma family of income distributions. Statistical distributions in scientific work, 6, 181-192.

Wilfling, B., Kramer, W. (1993). Lorenz ordering of Singh-Maddala income distributions. Econom. Lett. 43, 53-57.

Wilfling, B. (1996). Lorenz ordering of generalized Beta II income distributions. J Econometrics, 71, 381-388.

Zenga, M. (2007). Inequality curve and inequality index based on the ratios between lower and upper arithmetic means. Statistica \& Applicazioni, 5, 3-28.

Zenga, M. (2010). Mixture of Polisicchio's truncated Pareto distributions with beta weights. Statistica \& Applicazioni, 8, 3-25.

Zenga, M., Polisicchio, M., Zenga, Ma., Pasquazzi, L. (2011). More on M.M. Zenga's new three parameters distribution for non-negative variables. Statistica \& Applicazioni, 9, 5-33. 